\title{
Quo ante. Die natürliche Resilienz gegenüber radikalen Veränderungen und die digitale Transformation
}

\section{1 - Institutionelle Resilienz}

Der tertiäre Bildungsbereich, Universitäten und Hochschulen aller Art, bereitet junge Menschen auf ihr künftiges Leben, insbesondere ihr berufliches, in der bürgerlichen Gesellschaft und ihre Verwendung darin vor. Das war seine offizielle Aufgabe, seit dieser Bereich in verschiedenen Formen, dem Wesen aber gleich, seit dem Beginn aller staatlich organisierten Zivilisation institutionell eingerichtet, auskömmlich finanziert und auf absehbare Dauer gewährleistet worden ist: die Begründung seiner Existenz. Akademische Expertinnen und Experten, Literate, waren zunächst und sind bis heute für die Tradierung der religiösen Überlieferung gefragt. Die fortschreitende Ausdifferenzierung der wirtschaftlichen und kulturellen Reproduktion führte rasch zu weiteren Angeboten tertiärer Expertenbildung. Die Humboldtsche Idee tertiärer Bildung (Gall 2011, 162-65) machte von dieser Zwecksetzung, wie oft fälschlich unterstellt wird, keine Ausnahme. Humboldt sorgte mit seiner Modell-Universitätsgründung von 1809 nur für effektivere Methoden und organisatorische Rahmungen im Horizont seiner Gegenwart. Hochschulen verdanken ihre externe Zwecksetzung der pragmatischen Zukunftsvorbereitung, und sie müssen sich beständig fragen lassen, wie sie diesem Auftrag gerecht werden.

Die grundsätzliche Schwierigkeit, ja: fast eine Aporie dieser Konstellation, eine auf die Zukunft gerichtete Zwecksetzung in ein didaktisches Handeln in der Gegenwart zu überführen, gleichsam ins Blaue zu handeln, wird gerne mit einem abstrakten Verweis auf das Potential akademischer Freiheit in einem System verantwortlicher Selbststeuerung zwar rhetorisch überbrückt, aber in der Sache nur unzureichend adressiert. Für die Belange universitärer Forschung, die per definitionem, jedenfalls ihrer Idee nach, in ihrem Ausgang offen ist, mag das eine gute Antwort sein. Für den Bereich der akademischen Lehre hingegen mit seinem Apparat von Reglementen, kanonischen Vorgaben, Prüfungen, 
Evaluationen reicht dieser Verweis nicht, hat gewiss nie gereicht. Die vielbeschworene Einheit von Forschung und Lehre war auch an der Berliner Universität ein spannungsreiches Handlungsideal, insofern die Schulen, Kirchen, Kliniken, Verwaltungen von ihren spezifischen Anforderungen an die Personalakquise niemals lassen mochten (und zur Sicherheit externe Staatsexamina zu deren Prüfung verbindlich machten).

Die strukturelle Problematik folgt in sozialer Hinsicht aus der Tatsache, dass Dozierende Hochschulehre 'geben', Studierende also zu belehren unternehmen, Lehrveranstaltungen konzipieren, Lehrmittel produzieren, Studien- und Vorlesungspläne entwerfen und setzen und über das Mediensetting entscheiden, Studierendenleistungen bewerten, die selbst, jedenfalls zum grossen Teil und das insbesondere, wenn sie eine unbefristete Position erreicht haben, nicht in der langen Zukunft leben werden, auf die sie ihre Studierenden vorbereiten sollen, deren Erwartungshorizont bezüglich ihrer eigenen domänenspezifischen Praxis somit erheblich abweichen muss von dem ihrer Studierenden und deren Erfahrungsraum deutlich andersartige Prägungen hinterlassen hat (Koselleck 1976).

Das ist selbstverständlich ein durchaus allgemeines pädagogisches Strukturproblem, die Lebenserwartungsdifferenz der heutigen $\mathrm{Zu}$ kunftsagentinnen und -agenten und der späteren Zukunftsakteurinnen und -akteure, die wesentliche Phasenverschiebung der Horizonte zuungunsten der steuernden Akteure im Prozess des Unterrichts, instruktivistisch vereinseitigend 'Lehre' genannt.

Oftmals wird hier eingewendet, dass diese Lebenszeit-Differenz kein Problem, sondern vielmehr Charakteristikum jeder didaktischen Situation sei, und sie allererst zu einer a-symmetrischen Wissensaustauschsituation mache. Es ist allerdings nicht die Lebenszeit-Differenz als solche, die diese Situation konstituiert, sondern die Differenz von Wissen und methodischem Vermögen im Hinblick auf einen bestimmten Inhalt oder eine bestimmte Aufgabe. Für diese Differenz ist das Lebensalter der Beteiligten kein entscheidender Faktor. Jede Volkshochschule führt dies beständig vor, um nur ein Beispiel zu geben.

Im Rahmen universitärer Lehre tritt dies in der Regel aus der Wahrnehmung, weil insbesondere im deutschsprachigen Hochschulwesen mit seinen spezifischen Karrierebedingungen die Wissenshierarchieausübung besonders starr lebensaltersbezogen aufgefächert ist. Sie ist in diesem Sektor gesellschaftlicher Wissensreproduktion eine Normalität. Als Normalität wird sie in den Augen der Akteure unsichtbar, solange die für diese Normalität und in dieser Normalität geschaffenen Handlungs- 
rahmen stabil bleiben, woran wiederum jeder in ihnen bürgerlich etablierte Akteur sein Interesse hat. Unbefristet beschäftigte Dozierende sind mithin strukturell Normalitätsprofiteure.

Im Falle der digitalen Transformation, die zu reflektieren den Anlass dieses Textes bildet, spitzt sich dieses Strukturproblem der Lebenserwartungsdifferenz der Zukunftsagenten und der Zukunftsakteure erheblich zu. Die Erfahrungsdifferenz des Grossteils der heute unbefristet beschäftigten Dozierenden an den Hochschulen im Gegenüber zu ihren Studierenden ist in bestimmter Hinsicht, aber auch zugleich in allgemeiner: erheblicher, tiefgreifender, umfassender als sie es früher im Gegenüber anderer Generationen war. Es kann zugestandenermassen gar keinen Zweifel daran geben, dass es in der Vergangenheit auch schon ausserordentlich grosse Differenzen zwischen den Zukunftsagentinnen und -agenten und den Zukunftsakteurinnen und -akteuren gab, insbesondere in der Neuzeit, während der Jahrzehnte der industriellen Revolutionen (Osterhammel 2016, 909-957) oder auch in der Zeit des Durchbruchs des Wohlfahrtsstaates in den 1960er Jahren (Frei 2008).

Die Erfahrungsraumdifferenz zwischen den älteren Generationen, die ihre Primärsozialisation in den deutschsprachigen Ländern in einem Zeitalter der Nicht-Digitalität erfahren haben (ungefähr bis zum Geburtsjahrgang 1990, in regionaler Differenz) und denen, die in ihrer späten Pubertät und Adoleszenz von einem digitalen Alltag massgeblich bestimmt worden sind, ist immens. Zur Grenzbestimmung kann man sich auf die Markteinführung des iPhones im Jahre 2007 beziehen, die das WWW und zum technologischen Kern aller Jugendkultur ubiquitär machte, denn bald zogen billigere Konkurrenten nach. Die $1991 \mathrm{Ge}-$ borenen waren 200716 Jahre alt, also in einem Alter, in dem man selbstständige Kauf- und Kulturentscheidungen spätestens vorzunehmen vermag. Es liesse sich gewiss gut argumentieren, dass diese Generationenkluft zwischen den im Analogozän sozialisierten Menschen und den Angehörigen des Digitalozäns schon früher aufzusuchen ist. Netzarbeitern wie dem Autor und wahrscheinlich der geneigten Leserin oder dem Leser, war die digitale Arbeit schon seit Mitte der 90er Jahre alltäglich. Vielleicht ist aber die späte, konservative Epochenannahme, die auf die Ubiquität des Netzzugangs abhebt, verlässlicher, wenn es um Standardbestimmungen geht.

Über die materiale Differenz von Analogozän und Digitalozän muss hier vielleicht nicht viel geschrieben werden, man dekliniere nur die Bereiche der eigenen Lebenspraxis, um die Differenzen zu markieren. Dabei befindet sich die die digitale Transformation seit Jahren in expo- 
nentieller Beschleunigung (King 2016, 19 f.), 'Industrie 4.0' ist nicht nur ein Schlagwort, sondern eine Sammelbezeichnung für die Wirksamwerdung der Digitalisierung auf neuer Ebene, eine zweite digital getriebene industrielle Revolution (Deutsches Bundesministerium für Bildung und Forschung 2013). Die sich beschleunigende Transformation erweitert die mögliche Veränderungswahrnehmung zusehends ins Totale.

Marc Prensky (2001) hat schon sehr früh diese Unterscheidung der im Analogozän Sozialisierten von den später geborenen mit der Differenz von 'Digital Natives' und 'Digital Immigrants' zu fassen versucht, dabei aber bei den Natives gegenüber den Immigrants gleichsam natürliche Vorteile zu erkennen geglaubt. Prenskys Unterscheidung ist inzwischen vielfach und plausibel relativiert worden. Man kann dieses Begriffspaar auch wertfrei nutzen, ohne die Vorannahmen höherer digitaler Literarität unter den Natives. Ich kenne keine kürzere und prägnantere Bezeichnung, deshalb möchte ich dies gern, die Kritik an Prensky aufnehmend, ohne weitere Vorannahmen tun. Den Einwand, dass hinter dieser Differenzierung unterschiedliche ökonomische, soziale, kulturelle $\mathrm{Zu}$ gangschancen zur digitalen Transformation verborgen bleiben, ist richtig. Es berührt aber nicht die hier vorliegende Diskussion (Wampfler 2014).

Für die überwältigende Mehrheit der Absolvierenden von universitären Studienprogrammen wird diese kurze Zukunft der Dozierenden ausgesprochen lange dauern. Darin liegt ein Grund, warum Hochschulen, ihre Arbeits- und Unterrichtsmethoden und ihre Bildungsinhalte (ob Gegenstands- oder Handlungswissen) offenkundig als in Richtung bestenfalls ihrer Gegenwart, eigentlich aber in Richtung einer schon gestaffelten Vergangenheit von Zwecken und Normen orientiert erscheinen.

Unbefristete Dozierende bleiben lange im Amt, schöpfen nolens volens selbst aus einer persönlich erfahrenen akademischen Praxis, die vergleichsweise weit zurückreicht und sehr stark von beruflichen Alltagsroutinen und Traditionen bestimmt ist, tief verwurzelt im analogen Zeitalter, das gilt vor allem für die Kultur- und Geisteswissenschaften.

Die disziplinäre Studien-Grundlagenliteratur wurde zwar längst immerhin E-Learning und -plattformkompatibel gescannt. In der Regel handelt es sich dabei aber lediglich um Scan-Reproduktionen mit dem einzigen Mehrwert der leichteren Verfügbarkeit. Die Arbeitsweise in den Seminaren und Vorlesungen veränderte sich dadurch nur unbeträchtlich.

Universitäre Lehrmittel, v. a. lehrveranstaltungsorientierte Handbücher und besonders die erfolgreichen Longseller, verfasst wiederum von 
arrivierten Dozierenden mit langer akademischer Institutionenprägung, werden von den Verlagen aus guten betriebswirtschaftlichen Gründen noch immer vorwiegend als Printprodukte angeboten. Auch in Umgang mit diesen Eckpfeilern universitärer Lehrpraxis manifestiert sich die digitale Transformation lediglich als ein Verlust an Körperlichkeit und Gegenständlichkeit des Wissens. Die Lehrmittel erscheinen heutigen Studierenden im Kern in gleicher Form wie vor 25 Jahren, sie sind eben nur verbreitungseffektiv gescannt und in Universitätsclouds abrufbar abgelegt. Der Wissenshorizont dieser Lehrmittel ist allerdings ebenfalls notwendig auf die Gegenwart, in der Regel aber an den Horizonten einer gestaffelten Vergangenheit ausgerichtet. Das alles atmet und manifestiert den akademischen status quo ante.

Das ist ein strukturelles Problem von Bildungseinrichtungen, es tritt nur in einer Gegenwart der umfassenden digitalen Transformation besonders deutlich vor Augen. Hochschulen lehren nicht für die Zukunft, sondern für die vergangene Gegenwart von Menschen wie die des Autors und vermutlich der meisten der Leserinnen und Leser dieses Textes, die wir das Meiste unserer Biographien bereits gesehen haben.

Die Welt unserer Gegenwart ändert sich rasant. Man darf vermuten, dass an dieser Stelle der Einwand inzwischen mit Nachdruck erhoben wird: Was ist daran wirklich neu?

Natürlich gab es auch zu anderen Zeiten rasante Veränderungen. Vor allem das 20. Jahrhundert hat durch seine politischen und ideologischen Krämpfe, seine Kriege, Massenmigrationen, seinen Massenterror, die willkürliche Verschiebung von Grenzen usw. zu so vielen schnellen und umwälzenden Veränderungen in fast der ganzen Welt geführt, dass sich nach dem Ende des Kalten Krieges wahrscheinlich alle Menschen ein kommendes Zeitalter des Friedens, der Stabilität, der Sicherheit und der kulturellen Kontinuität im Wohlstand und der individuellen Freiheit wünschten. So unterschiedlich die Situationen in unseren Ländern auch gewesen sein mögen, dieser Wunsch hat wahrscheinlich die überwiegende Mehrheit der damaligen Menschen bewegt, und wie oft und einschneidend wurde diese Hoffnung seitdem enttäuscht. Kann etwas nicht einfach so bleiben, wie es ist, wenn es gut oder zumindest erträglich ist? Das ist eine Frage, die viele zu bewegen scheint, jedenfalls wäre das eine mögliche Erklärung für das Erstarken identitärer und populistischer politischer Bewegungen.

Vielleicht ist es dieser Kontext, die effektive lange Erfahrung der zwei oder drei aktiven Generationen, die es für Schulen und Universitäten besonders schwierig macht, schnelle und adäquate Lösungen für eine 
neue superschnelle Transformation der Welt zu finden und ihr in der akademischen Bildungsrealität Geltung zu verschaffen, um die es jetzt im Folgenden gehen soll: der digitalen Transformation, der zeitversetzten Disruption aller Gewohnheiten, zeitlich gestaffelt je nach Beharrungskraft des gesellschaftlichen Subsystems, nach dem Muster des Dominoprinzips.

Bevor es jedoch zu einer Differenzierung dieser digitalen Herausforderung speziell des Systems der tertiären Lehre kommt, soll auf zwei weitere Faktoren hingewiesen werden, die einer angemessen raschen und tiefgreifenden Veränderung und Anpassung des Hochschulsystems im Wege stehen.

Die Grundfaktoren des allgemeinen Hochschulsystems, seine Gebäude und deren Struktur, deren Einrichtung, die Unterrichtsformen, die Erwartungen an Dozierende und Studierende, seine kanonischen Inhalte sind in den letzten sicher 50 Jahren, sektoral aber länger, mehr oder weniger unberührt geblieben. Nur die dafür gesetzten Ziele und einige pädagogische Ideen haben sich etwas verändert, Inhaltselemente wurden gestrichen oder ergänzt. Aber es ist jedenfalls seit der Bildungsexpansion Ende der 60er/Anfang der 70er Jahre bis heute mehr oder weniger das gleiche Spiel geblieben, so wie sich der Fußball entwickelt hat, aber immer noch Fußball ist. Diese Art von Hochschule wird heute durch die umfassende digitale Revolution grundlegend in Frage gestellt. Niemand konnte darauf vorbereitet sein oder darauf vorbereitet werden. Das Ausmass der nötigen personalen, organisatorischen und baulichen Veränderungen erscheint unabsehbar gross, die seit 20 Jahren immer wieder in Teilbereichen administrativ erzwungenen Anpassungen erschienen defensiv, oft ohne gestalterische Leitidee, ohne Vision und Einsicht in einen Gesamtprozess, getragen nur von wenigen Spezialisten und haben kaum einen Dozierenden als Zuwachs an Arbeitsqualität überzeugt. Nach der Stabilisierung der anfänglich auch noch lange Zeit wenig funktionalen Lehrverwaltungs- und E-Learning-Tools (zur Kritik vgl. Demantowsky 2015), vermag man immerhin Gewöhnungseffekte an einen Stand von digitaler Technologie festzustellen, den es schon seit mindestens 15 Jahren gibt, der inzwischen allerdings einfach besser funktioniert.

Diese halbherzigen und oftmals ungeeigneten Versuche der universitären Verwaltungen angemessen zu reagieren, oftmals nur motiviert und durch die Geldgeber angeschoben im Blick auf Einsparpotentiale von Personalkosten, haben eine grundsätzliche Abwehrhaltung, eine Resilienz verstärkt, deren Hauptanliegen es ist, die gelernten Routinen der tertiären Lehre zu bewahren und die gesellschaftlich längst tiefgreifend 
sichtbar werdende digitale Transformation so lange als möglich vom behüteten Raum der eigenen Berufspraxis fernzuhalten.

Darüber hinaus gibt es ein weiteres, vielleicht unerwartetes Problem: die zunehmende Attraktivität gewinnorientierter technologischer Angebote großer digitaler Unternehmen, wie sie sich auf den grossen Bildungs- und Game-Messen der Welt schon jetzt in erstaunenswerter Weise betrachten lassen. Je grösser die Differenz zwischen dem Stand der gesellschaftlichen digitalen Transformation und der akademischen Lehrrealität, zwischen den Bedürfnissen einer dominant digital geprägten Generation und den älteren Dozierenden, zwischen den Anforderungen einer antizipierbaren späteren Berufsrealität und den tertiären Lehrangeboten wird, desto grösser wird die Chance und Wahrscheinlichkeit, dass die großen digitalen Konzerne nun auf breiter Front in dieses Feld eintreten und die Hochschulen in Instrumente der Kundenbindung und Datenproliferation verwandeln. Dieser Prozess lässt sich an den Sekundarschulen schon jetzt beobachten. Viele Lehrpersonen kritisieren dies zu Recht. Es nährt zusätzlich ihre Skepsis, ihre Lehrkonzepte und Lehrhabitus angesichts der digitalen Transformation zu überdenken. Digitale Resilienz wird so zu einem Aspekt von kapitalismusskeptischer Gesellschaftskritik, was die Realität dann richtig spiegelt, aber an den Herausforderungen und Chancen der digitalen Transformation nicht zuletzt auch für die akademische Lehre vorbeigeht.

Schliesslich, um einen letzten Aspekt für die dominierende digitale Resilienz des akademischen Lehrbetriebs anzuführen, das fortbestehende Orientieren an einem Status quo ante: Es fehlt an praktisch erprobten und wissenschaftlich getesteten hochschuldidaktischen Modellen für die digital fundierte und orientierte tertiäre Lehre in den einzelnen Disziplinen. Es fehlt an disziplinspezifischen Operationalisierungen. Nur sehr wenige Kolleginnen und Kollegen haben sich um die vorhersehbaren Herausforderungen der digitalen Transformation gekümmert; nur sehr wenige digital aufgeschlossene und teils digital begeisterte Dozierende konnten Konzepte und Ideen an der Basis entwickeln (vgl. Demantowsky 2017). Die wenigen dieser Bemühungen blieben weitgehend isoliert, fanden kaum Anschluss und Nachahmung. Auch die elaborierte medienpädagogische Forschung mit ihren speziellen Lehrstühlen und Abteilungen (vgl. exzellent: Petko 2014) blieb weitgehend ein geschlossener Bereich. Seit wenigen Jahren zeigen sich hier insofern Veränderungen, als die Nachfrage nach entsprechenden Fortbildungen steigt. Fakultätsleitungen erkennen den Bedarf, zunehmend mehr Dozierende folgen den Einladungen in diese Veranstaltungen. 
Seit wenigen Jahren entwickelt sich, auch das ein Hoffnungszeichen, in den fachspezifischen disziplinären Didaktiken eine Forschungslandschaft, die vor allem von jungen Doktorierenden geprägt wird, deren Lebensrealität das digitale Eintauchen einschließt; sie gehören zu den Digital Natives (Prensky 2001).

\section{2 - Was bedeutet die digitale Transformation für die tertiäre Lehre}

Aber was ist diese digitale Transformation, die in vielerlei Hinsicht auch als Revolution bezeichnet werden kann? Diese Frage kann auf viele Arten diskutiert werden, aber hier soll nur ein Aspekt kurz angerissen und dann anhand von sechs Feldern beispielhaft verdeutlicht werden, der für das Hochschulsystem besonders relevant ist, nämlich die völlig differente Art und Weise, wie im Vergleich zum Analogozän Wissen in allen Bereichen dokumentiert, kontextualisiert, gespeichert, strukturiert und präsentiert wird oder werden kann.

Das Konzept des Digitalen ist, wenigstens als Wort, als Bezeichnung, inzwischen mehr als vertraut. Es klingt abstrakt, kaum noch jemand denkt darüber nach. Dennoch beschreibt es sehr genau, was sich alles und wie es sich verändert. Die Tatsache, dass Informationen binär kodiert sind, ist nur scheinbar ein gradueller Unterschied zu komplexeren Kodierungen, wie beispielsweise der tausendfachen Leistung menschlicher Sprachen und ihrer Zeichensysteme. Die radikale Einfachheit dieser binären 1-0Codierung lässt buchstäblich keinen Stein unverrückt, stellt alle Gewissheiten in Frage. Wissen unterschiedlichster Herkunft und Codierung verwandelt sich, und es funktioniert eigentlich fast wie die Alchemie, sowohl hinsichtlich der Neu-Erzeugung von Objekten unseres Wissens als auch der Form des Wissens über diese Objekte. Die radikal einfache Binärcodierung formiert schliesslich beides digital fortschreitend zur Vollständigkeit. Das Attribut 'digital' ist damit technisch konkret, auch wenn die daraus resultierende Vielfalt von Format, Anwendung, Komplexität und Praxis universell ist. Das ist das Paradoxon dieses Konzepts des Digitalen: Extrem konkret und extrem allgemein zugleich zu sein. Die einfachste Codierung macht jede Information nahezu beliebig übertragbar, lesbar, speicherbar, formbar, übersetzbar, verknüpfbar, durchsuchbar, ausbeutbar. Die tendenziell komplette Vernetzung diverser Daten planiert Gebirge von Inkommensurabilität jeder Art. 
Sechs technologische Innovationen aus dieser so begriffenen Digitalität verändern unsere Weltbegegnung und damit auch alle Bildungsprozesse, ob intentional oder non-intentional, grundlegend. Nicht überall auf der Welt zur gleichen Zeit selbstverständlich, sondern vielmehr ausgehend von Regionen, Bereichen, Gruppen mit hoher Innovationsdichte allmählich in alle anderen Regionen, Bereiche, Gruppen in einer Geschwindigkeit, die vom Verhältnis der Investitionskosten zu den bereitgestellten Ressourcen abhängt sowie von Profitantizipationen und ihrer Marktfreiheit.

Einige kurze Bemerkungen zu diesen sechs technologischen Innovationen in ihrer beobachtbaren oder antizipierbaren Rückwirkung auf tertiäre Lehre.

\section{Die Speicher- und Netzrevolution}

Das Wissen aller möglichen Formen ist nicht mehr in geschützten Institutionen mit eingeschränktem (zumindest kanalisiertem, kontrolliertem) Zugang konzentriert. Jede Person und jeder Studierende hat permanenten und ubiquitären Zugang zur größten Bibliothek aller Zeiten, insofern diese in der Regel beständig in der Hosentasche getragen wird. Man braucht nicht in die nächstgrößere Stadt oder ins Stadtzentrum zu fahren. Man muss nur noch verschwindend wenig Zeit, Geld, Energie investieren, um an Informationen zu gelangen. Es ist alles einfach da, in jeder Hosentasche, solange man sich in der Reichweite eines Telefonnetzes befindet. Darüber hinaus werden diese Wissensressourcen als Ganzes nur mit hohem technischen Aufwand geschlossen, können jedenfalls nicht verbrannt werden, können nur sehr mühsam indiziert werden (wiederum abhängig von spezifischen Orten in einer globalen Infrastruktur des Wissens) - denn als multiple dezentrale Informationen unterliegen sie nicht den lokalen Bedingungen eines Ortes. Aber nun, wo ist die Hochschuldidaktik des allgemein zugänglichen Wissens für alle Fachgebiete? Wo ist die didaktische Reflexion auf die Einebnung von Wissenshierarchien zwischen Dozierenden und Studierenden? Wir arbeiten immer noch überwiegend mit Lehrbüchern oder ihren Zwillingsgeschwistern: den Moodle-Kursen und damit auch deren heteronomen Heuristiken und deren Gesten des Ausreichendseins. 


\section{Games und Gamification}

Die Game-Industrie gilt heute als einer der wichtigsten Inkubatoren für technologische Innovationen. Wer die führenden Spielemessen besucht, besucht die mittelfristige Zukunft der epistemischen Reproduktion. Über die Transponierung der effektiven Strukturen digitaler Games in alle denkbaren Bereiche von Wirtschaft, Militär, Gesellschaft, die Gamification, werden die Entwicklung der Game-Industrie allerorts wirksam (Anderie 2018, 37-41).

Viele junge Menschen verbringen heute einen relevanten Teil ihrer Freizeit in digitalen Spielwelten. Wer wissen will, welche Präkonzepte beziehungsweise Alltagstheorien viele Studierenden in die Lehrveranstaltungen mitbringen, der muss eine der gegenwärtigen Hauptquellen intensiv betrachten und deren sehr prägnante, digital generierte imaginäre Welt kennen. Gleiches gilt für Streaming-Dienste mit ihren grenzenlosen Filmserien. Beide Verbraucherangebote von Ideen, Bildern, Klängen, Gefühlen, Vorstellungen, d.h. kodiertem Wissen fast aller Art, werden von den Spielerinnen und Spielern umfassend aufgenommen und sind daher für die disziplinäre akademische Lehre relevant, weil sie sich äußerst unauffällig und dennoch effektiv auf die neuropsychologische Natur des menschlichen Gehirns beziehen. Auch die taktilen Empfindungen werden eine Rolle bekommen. (Björn Klein weist darauf hin: sie haben es längst. Man denke an die DualShock Gamescontroller, die taktile Empfindungen schon seit 1998 imitieren.)

Die Game-Programmierungen arbeiten virtuos mit Belohnungen, mit emotionalen Triggern und befinden sich nun auf einer technischen Ebene, die Erfahrungswelten bietet, mit denen das wirkliche Leben oft kaum mithalten kann. Die Lehranstrengungen zur Schaffung und Aufrechterhaltung der Motivation tun gut daran, diese Muster zu reflektieren, sich mindestens damit auseinanderzusetzen. Man kann sehr viel und sehr intensiv lernen über Physik, Geschichte, Musik oder Biologie, die Frage ist, in welchem Verhältnis dieses Wissen zu wissenschaftlich begründetem Wissen steht oder zu stehen kommt.

\section{Mixed Reality}

Mixed Reality entführt all jene, die sich ihr aussetzen, buchstäblich in eine andere Welt. Diese Reise wird immer noch durch die Unhandlichkeit der entsprechenden Geräte, zumeist einer Art von Brille, behindert, aber das 
Bequemerwerden ist nur eine Frage der technischen Entwicklung. In dieser Mixed Reality sind Informationen über die dort zu sehende Welt in die Objekte eingewoben. Wir müssen Informationen nicht mehr auf einem Computerbildschirm abrufen. Es ist das Weltwissen oder eben die Auswahl der Gestalter omnipräsent, inhärent den Gegenständen der immersiven digitalen. Wissen begegnet den Nutzenden, Akteursbewusstsein wird radikal in Frage gestellt. Die Realität der Nutzerinnen und Nutzer wird buchstäblich erweitert. Ob die Informationen korrekt sind, welche Perspektiven sie enthalten, wer die Autorinnen oder Autoren sind, ist für User nur schwer zu verstehen, ganz anders als bei einem herkömmlichen Lehrbuch. Mixed Reality enthält auch potenziell unüberschaubare Elemente der virtuellen Realität. Die Annahme einer für die menschliche Erfahrung konstitutiven Übereinstimmung der tatsächlichen Objekte in der Welt wird abgeschafft. Korrespondenz besteht nur zu den Objekten innerhalb dieser Virtualität, für die uns die Erfahrung der Beständigkeit fehlt. Wenn man sich jetzt die Augmented mit der virtuellen Realität ergänzt vorstellt, dann betrachtet man diese harmlos klingenden Mischrealitäten hier in diesem Text noch von aussen. Tatsächlich überschreibt Mixed Reality jedoch alle epistemischen Gewissheiten der bisherigen akademischen Schulweisheit. Wo sind die neue Hermeneutik und Ontologie der gemischten Welten?

\section{Granulation via Algorithmisierung}

Die Welt, wie wir sie kennen, ist auf Standard getrimmt (sehr eingängig: Gehlen 2017). Die Geschichte der Moderne ist eine Geschichte der Standardisierung der Welt. Die Entwicklung der modernen Universität in Europa im 19. Jahrhundert und auch die tertiäre Bildungsexpansion vor 50 Jahren selbst sind Instrumente der Standardisierung. Diese Normen haben die Industrie ermöglicht, Bildung für alle, Tourismus, Fernsehen usw., aber dieses System hat auch viele Nachteile, denn es passt nie ganz zu uns, was für uns qua Norm zugedacht ist, manchmal passt es auch gar nicht. Auch hier führt die digitale Transformation zu einer Umkehrung aller Gewohnheiten, paradoxerweise durch die Radikalisierung der Standardisierung von Informationssignalen ins Binäre. Dies und die Speicherrevolution ermöglichen es, enorme Datenmengen algorithmisch zu verarbeiten und verfügbar zu halten, die es dann ermöglichen und sinnvoll machen, die Angebote zu granulieren, d. h. sie im Detail an die Bedürfnisse des Einzelnen anzupassen. Das ist etwas, was 
man schon heute in den neuesten Infrastruktursystemen beobachten kann, versteckt in vielen Stellen des Alltags ist es bereits Realität, wenn auch vielleicht seltener in Mitteleuropa. Was das für die tertiäre Lehre bedeutet, auch in Verbindung mit der Gamification, wird ohne weiteres deutlich: das absehbare Ende des traditionellen hierarchischen Unterrichts in grossen Gruppen zugunsten individualisierter Lernumgebungen und personaler Curricula.

\section{Robotics}

Wir wissen, dass menschliche Arbeit schrittweise durch Maschinen ersetzt wird, seitdem es die menschliche Kultur gibt. Neu ist wahrscheinlich, dass dieser Ersatz in Bereiche vordringt, in denen die Menschen bisher dachten, dass sie vor dem Austausch sicher wären, z. B. in der Universität. Man stelle sich vor, es würde Robotik mit Gamification, Mixed Reality und Granulation verbunden, und man sieht, dass solche intelligenten Maschinen neue Lernumgebungen in einer Perspektive schaffen können, die auf den einzelnen Studierenden zugeschnitten sind, die nicht müde oder unprofessionell werden und absolut neutral gegenüber jedem Lernenden sein können, insofern ihre interne interaktive Wissensbegegnungssteuerung über abstrakte Algorithmen und konkrete individuelle Dateninputs und -outputs erfolgt. Das geteilte und durch Designer intentional gestaltete Interface stellt demgegenüber nur noch die Benutzungsoberfläche dar. Robotics funktionieren evidenzbasiert über rückgekoppelte Testverfahren. Darüber hinaus werden ihre Anschaffung und Instandhaltung mittelfristig weitaus günstiger sein als die Beschäftigung eines Menschen. Sie werden kommen (Bodkin 2017).

\section{Visual Perceptive Media}

Eine Amalgamierung all dieser Technologien mündet in die Visual Perceptive Media. Nur ein kurzer Blick darauf: Diese Technologie an der Schnittstelle von Mensch und Maschine betrifft ein Grundverhältnis moderner Gesellschaften, nämlich dass grundsätzlich alle Mitglieder einer Gesellschaft die gleichen kulturellen Erfahrungen in medialen Umgebungen haben oder zumindest haben können. Diese grundlegende Beziehung bestimmt auch weitgehend unsere Bildungssysteme, sie regelt den Status von Studienplänen und Lernmitteln. Aber darüber hinaus ist es 
für unser Selbstbild im Kollektiv unerlässlich, dass wir uns selbst erkennen, indem wir die gleichen Filme gesehen, die gleichen Spiele gespielt, die gleiche Musik gehört haben. Visual Perceptive Media verändern das Medienangebot je nach Nutzer, je nach Zustand, Stimmung. Sie sitzen nebeneinander und sehen einen anderen Film, eine andere Handlung, eine andere Kulisse. Wir verlassen die gemeinsame Realität eines Kollektivs. Welche Antworten hätte ein Studium künftiger kultureller Multiplikatoren darauf? (BBC abger. 2019)

\section{$3-$ Was ist zu tun?}

Wenn das System tertiärer Bildung an den Hochschulen und Universitäten weiterhin die Vorbereitung auf eine lange Zukunft seiner Studierenden übernehmen oder sogar sicherstellen soll, dann müssen alle Gewohnheiten und Einrichtungen dieser akademischen Institutionen auf den Prüfstand gestellt werden. Eine große Reformdebatte ohne Tabus müsste beginnen. Hochschuldigitaldidaktische Labore müssten überall eröffnet werden, jedes mit einem anderen Akzent, die eingespielten und selbst routinisierten Kreise der Experten und Expertinnen, zu denen sich auch der Autor selbstkritisch zählt, müssten aufgebrochen und durch Interessenten jeden Alters und Berufes, aller sozialen Milieus ergänzt werden. Dozierende müssten wieder Studierende werden wollen, der Staat oder der jeweilige finanzielle Träger müsste bereit sein, viel Geld zu investieren und sich dabei an der Idee der Subsidiarität zur Gewährleistung von freier und aufs Ganze bedachter Konkurrenz orientieren. Gleichzeitig sollte empirisch systematisch untersucht werden, wie die Hochschule langfristig in einer post-digitalen Gesellschaft Studierende bewusst befähigen kann, das Andere, die Magie und die Grenzen des Analogen zu erfahren.

Die Welt um uns herum verändert sich in der digitalen Transformation rasant, und wenn sich die tertiäre Lehre als System nicht proaktiv und visionär gestaltend an sie anpasst, verliert sie einen wichtigen Teil ihrer gesellschaftlichen Funktion: die Vorbereitung junger Menschen auf die Zukunft. 


\section{Referenzen}

Anderie, Lutz: Gamification, Digitalisierung und Industrie 4.0. Transformation und Disruption verstehen und erfolgreich managen. Wiesbaden 2018.

BBC, Research \& Development: Visual Perseptive Media. Personalised video which responds to your personality and preferences. Online: https://www. bbc.co.uk/rd/projects/visual-perceptive-media [abgerufen: 6. Juni 2019].

Bodkin, Henry: 'Inspirational Robots' to begin replacing teachers within 10 years. In: The Telegraph (11. September 2017), Online: https://www.tele graph.co.uk/science/2017/09/11/inspirational-robots-begin-replacing-tea chers-within-10-years/ [abgerufen: 6. Juni 2019].

Deutsches Bundesministerium für Bildung und Forschung. Referat IT-Systeme: Zukunftsbild „Industrie 4.0“. Bonn 2013.

Demantowsky, Marko: Die Geschichtsdidaktik und die digitale Welt. Eine Perspektive auf spezifische Chancen und Probleme. In: ders., \& Pallaske, Christoph (Hg.): Geschichte lernen im digitalen Wandel. München 2015, S. $149-161$.

Demantowsky, Marko: Wikipedia und Lehrerbildung. In: Forum Didaktik der Gesellschaftswissenschaften in der Nordwestschweiz (12. Oktober 2017), Online: http://www.gesellschaftswissenschaften-phfhnw.ch/wikipedia-und-lehrer bildung/, [abgerufen: 6. Juni 2019].

Frei, Norbert: 1968. Jugendrevolte und globaler Protest. München 2008.

Gall, Lothar: Wilhelm von Humboldt. Ein Preusse in der Welt. Berlin 2011.

Gehlen, Dirk v.: Meta! Das Ende des Standards. Berlin, 2017.

King, Brett: Augmented - Life in the smart lane. Singapore 2016.

Koselleck, Reinhart: „Erfahrungsraum“ und „Erwartungshorizont“ - zwei historische Kategorien [1976]. In: ders., Vergangene Zukunft. Zur Semantik geschichtlicher Zeiten. Frankfurt/Main 1989, S. 349-375.

Osterhammel, Jürgen: Die Verwandlung der Welt. Eine Geschichte des 19. Jahrhunderts. München 2016.

Petko, Dominik: Einführung in die Mediendidaktik. Lehren und Lernen in digitalen Medien. Weinheim 2014.

Prensky, Marc: Digital Natives, Digital Immigrants. In: On the horizon 9,5 (2001) S. $1-6$.

Wampfler, Philipp: Bitte verzichtet auf den Begriff „digital natives“! In: Schule Social Media (12. August 2014), Online: https://schulesocialmedia.com/ 2014/08/12/bitte-verzichtet-auf-den-begriff-digital-natives/ [abgerufen: 6. Juni 2019]. 